\title{
NOTE ON DEGREE OF TRIGONOMETRIC AND POLYNOMIAL APPROXIMATION TO AN ANALYTIC FUNCTION, IN THE SENSE OF LEAST $p$ TH POWERS
}

\author{
J. L. WALSH AND W. E. SEWELL
}

1. Introduction. In a recent note ${ }^{1}$ the present writers studied the relation between the continuity properties of a function and the degree of approximation in the sense of Tchebycheff by trigonometric and other polynomials; this approximation in the $z$-plane is considered either on the unit circle by polynomials in $z$ and $1 / z$, or on the segment $-1 \leqq z \leqq 1$ by polynomials in $z$, or (for functions of period $2 \pi$ ) on the infinite interval $-\infty<z<\infty$ by trigonometric polynomials. In the respective cases, the functions approximated are analytic in an annulus $\rho>|z|>1 / \rho<1$, in an ellipse whose foci are +1 and -1 , or in a horizontal strip containing the axis of reals in its interior. It is the purpose of the present note to establish the analogous results when approximation is measured by the integral of the $p$ th power of the error, as in the sense of least $p$ th powers.

The method we employ makes essential use of the specific results concerning Tchebycheff approximation as developed in our previous note, together with certain general methods already developed elsewhere. $^{2}$

2. Approximation on the unit circle. Our main result is as follows:

THEOREM 1. Let the weight function $w(\theta)$ be positive and continuous for all values of $\theta$, and of period $2 \pi$. Let the function $f(\theta)$ (not necessarily real) be periodic with period $2 \pi$, and suppose the numbers $a_{n j}$ and $b_{n j}$ (not necessarily real) are given so that

$$
s_{n}(\theta)=\frac{a_{n 0^{-}}}{2}+\sum_{j=1}^{n}\left(a_{n j} \cos j \theta+b_{n j} \sin j \theta\right),
$$

with the relation, for $n=1,2, \cdots$,

$$
\begin{aligned}
\int_{-\pi}^{\pi} w(\theta)\left|f(\theta)-s_{n}(\theta)\right| p d \theta \leqq & \frac{M}{\rho^{n p} n^{(k+\alpha+1) p}}, \\
& 0<\alpha \leqq 1, p>0, \rho>1,
\end{aligned}
$$

1 This Bulletin, vol. 44 (1938), pp. 865-873. We shall refer to this note as WS.

2 Walsh, Interpolation and Approximation by Rational Functions in the Complex Domain, American Mathematical Society Colloquium Publications, vol. 20, New York, 1935. 
where $k$ is a nonnegative integer and $M$ is a constant independent of $n$. Then the function

$$
\begin{aligned}
F(z) & =\lim _{n \rightarrow \infty}\left[c_{n 0}+\sum_{j=1}^{n}\left(c_{n,-j} z^{-j}+c_{n j} z^{j}\right)\right], \\
2 c_{n j} & =a_{n j}-i b_{n j}, \quad 2 c_{n,-j}=a_{n j}+i b_{n j},
\end{aligned}
$$

coincides with $f(\theta)$ almost everywhere (everywhere if $f(\theta)$ is continuous) on the circle $|z|=1$, with $z=\cos \theta+i \sin \theta$, and $F(z)$ is analytic in the annulus $\rho>|z|>1 / \rho$ and continuous in the corresponding closed region. For $z_{1}$ and $z_{2}$ on $|z|=\rho$ or $1 / \rho$ we have ${ }^{3}$

$$
\left|F^{(k)}\left(z_{1}\right)-F^{(k)}\left(z_{2}\right)\right| \leqq L\left|z_{1}-z_{2}\right|^{\alpha} \cdot|\log | z_{1}-\left.z_{2}\right|^{\beta}
$$

where $\beta=0$ if $\alpha<1$ and $\beta=1$ if $\alpha=1$, and where $L$ is a constant independent of $z_{1}$ and $z_{2}$.

As a matter of convenience in the proof of Theorem 1 we establish a preliminary proposition:

LEMMA. Let $C$ be a rectifiable Jordan curve in the z-plane containing $z=0$ in its interior; let $P(z, 1 / z)$ be a polynomial in $z$ and $1 / z$ of degree $n$ :

$$
\begin{aligned}
P(z, 1 / z) & \equiv P_{1}(z)+P_{2}(z), \\
P_{1}(z) & =a_{0}+a_{1} z+\cdots+a_{n} z^{n}, \\
P_{2}(z) & =a_{-1} z^{-1}+a_{-2} z^{-2}+\cdots+a_{-n} z^{-n},
\end{aligned}
$$

and suppose

$$
\int_{C}|P(z, 1 / z)| p|d z| \leqq N^{p}, \quad \quad p>0 .
$$

Denote by $w=f_{1}(z)$ a function which maps the exterior of $C$ onto $|w|>1$ so that $f_{1}(\infty)=\infty$, and denote by $w=f_{2}(z)$ a function which maps the interior of $C$ onto $|w|<1$ so that $f_{2}(0)=0$; denote by $C_{R}$ the closed annular region bounded by the two Jordan curves $\left|f_{1}(z)\right|=R>1,\left|f_{2}(z)\right|=1 / R$.

Then there exists a constant $N^{\prime}$ depending on $C, p$, and $R$ but not on $P(z, 1 / z)$ nor on $n$, such that (4) implies

$$
|P(z, 1 / z)| \leqq N N^{\prime} R^{n}, \quad z \text { in } C_{R} .
$$

By a known method of proof (Walsh, op. cit., p. 92) we have for suitably chosen $N^{\prime}$

${ }^{3}$ The notation $F^{(k)}(z)$ indicates the $k$ th derivative of $F(z)$ if $k>0$ and the function $F(z)$ itself for $k=0$. Here and below such derivatives on the boundaries of regions of analyticity are considered in the one-dimensional sense. 


$$
\begin{aligned}
& |P(z, 1 / z)| \leqq N N^{\prime} R^{n}, \quad z \text { on curve }\left|f_{1}(z)\right|=R \\
& |P(z, 1 / z)| \leqq N N^{\prime} R^{n}, \quad z \text { on curve }\left|f_{2}(z)\right|=1 / R .
\end{aligned}
$$

The lemma follows at once. ${ }^{4}$

We proceed now with the proof of Theorem 1. From (1) by the well known general in equalities

$$
\begin{array}{lr}
\left|\chi_{1}+\chi_{2}\right|^{p} \leqq 2^{p-1}\left|\chi_{1}\right|^{p}+2^{p-1}\left|\chi_{2}\right|^{p}, & p>1, \\
\left|\chi_{1}+\chi_{2}\right|^{p} \leqq\left|\chi_{1}\right|^{p}+\left|\chi_{2}\right|^{p}, & 0<p \leqq 1,
\end{array}
$$

and by virtue of the boundedness of $1 / w(\theta)$, we have

$$
\int_{-\pi}^{\pi}\left|s_{n+1}(\theta)-s_{n}(\theta)\right|^{p} d \theta \leqq \frac{M_{1}}{\rho^{n p} n^{(k+\alpha+1) p}} .
$$

On the circle $|z|=1$ we have $\cos j \theta=\left(z^{i}+z^{-j}\right) / 2, \sin j \theta=\left(z^{j}-z^{-i}\right) / 2 i$, so the first member of (6) can be written

$$
\int_{|z|=1}\left|P_{n+1}(z, 1 / z)-P_{n}(z, 1 / z)\right| p|d z|
$$

where $P_{n}(z, 1 / z)$ is a polynomial of degree $n$ in $z$ and $1 / z$, equal to $s_{n}(\theta)$ on $|z|=1$. From inequality (6) we now deduce through the lemma for $\rho \geqq|z| \geqq 1 / \rho$,

$$
\left|P_{n+1}(z, 1 / z)-P_{n}(z, 1 / z)\right| \leqq M_{2} / n^{k+\alpha+1},
$$

where $M_{2}$ is independent of $n$ and $z$. We define $F(z)$ for $\rho \geqq|z| \geqq 1 / \rho$ by means of the equation

$$
\begin{aligned}
F(z) \equiv P_{1}(z, 1 / z)+ & {\left[P_{2}(z, 1 / z)-P_{1}(z, 1 / z)\right] } \\
+ & {\left[P_{3}(z, 1 / z)-P_{2}(z, 1 / z)\right]+\cdots, }
\end{aligned}
$$

whence from ( 7$)$ we have for $\rho \geqq|z| \geqq 1 / \rho$

$$
\begin{aligned}
\left|F(z)-P_{n}(z, 1 / z)\right| \leqq & \left|P_{n+1}(z, 1 / z)-P_{n}(z, 1 / z)\right| \\
& +\left|P_{n+2}(z, 1 / z)-P_{n+1}(z, 1 / z)\right|+\cdots \\
\leqq & M_{2} \sum_{j=n}^{\infty} \frac{1}{j^{k+\alpha+1}} \leqq \frac{M_{3}}{n^{k+\alpha}}
\end{aligned}
$$

where $M_{3}$ is independent of $n$ and $z$.

The uniformity of the convergence in (8) is included in the inequalities (9), so it follows that $F(z)$ is analytic for $\rho>|z|>1 / \rho$, continuous

${ }^{4}$ So far as the writers are aware, this explicit lemma has not been previously formulated in print, but indications of it are given by Walsh, American Journal of Mathematics, vol. 54 (1932), pp. 559-570. 
for $\rho \geqq|z| \geqq 1 / \rho$; and since $P_{n}(z, 1 / z)$ on $|z|=\rho$ and $|z|=1 / \rho$ is a trigonometric polynomial of order $n$, it follows from a theorem due to de la Vallee Poussin ${ }^{5}$ that $F(z)$ satisfies an inequality with respect to $\theta$ of form (3), $z=\rho e^{i \theta}$ or $e^{i \theta} / \rho$, from which it follows that inequality (3) itself is fulfilled on $|z|=\rho$ and $|z|=1 / \rho$.

The boundedness of $1 / w(z)$ implies from (1)

$$
\int_{-\pi}^{\pi}\left|f(\theta)-s_{n}(\theta)\right| p d \theta \leqq \frac{M_{4}}{\rho^{n p} n^{(k+\alpha+1) p}} .
$$

Inequality (9) implies, from the equality of $P_{n}(z, 1 / z)$ and $s_{n}(\theta)$ on $|z|=1$,

$$
\int_{-\pi}^{\pi}\left|F(z)-s_{n}(\theta)\right| p d \theta \leqq \frac{M_{5}}{n^{(k+\alpha) p}},
$$

so we now deduce by use of (5) and by allowing $n$ to become infinite

$$
\int_{-\pi}^{\pi}|F(z)-f(\theta)| p d \theta=0
$$

hence $F(z)$ and $f(\theta)$ are equal almost everywhere on $|z|=1$, and Theorem 1 is established.

3. Approximation on the segment $-1 \leqq z \leqq 1$. We shall indicate rapidly the proof of the next theorem.

THEOREM 2. Let $f(z)$ be defined on the segment $-1 \leqq z \leqq 1$, and for $n=1,2, \cdots$ let a polynomial $P_{n}(z)$ in $z$ of degree $n$ exist such that

$$
\begin{aligned}
\int_{-1}^{1} w(z)\left|f(z)-P_{n}(z)\right| p d z \leqq & \frac{M}{\rho^{n p} n^{(k+\alpha+1) p}}, \\
& 0<\alpha \leqq 1, p>0, \rho>1,
\end{aligned}
$$

where $k$ is a nonnegative integer, and where $w(z)\left(1-z^{2}\right)^{1 / 2}$ is positive and continuous on $-1 \leqq z \leqq 1$. Then the function $f(z)$ is equal to $F(z)$ almost everywhere on the segment $-1 \leqq z \leqq 1$, where

$$
F(z)=\lim _{n \rightarrow \infty} P_{n}(z) \text {. }
$$

Furthermore $F(z)$ is analytic throughout the interior of the ellipse $\gamma$ whose foci are +1 and -1 and the sum of whose semi-axes is $\rho$; also $F(z)$ is continuous in the corresponding closed region, and satisfies an inequality of type (3) on $\gamma$.

\footnotetext{
${ }^{5}$ Leçons sur l'Approximation, Paris, 1919, chap. 4.
} 
We map the $z$-plane onto the w-plane by the transformation $z=\left(w+w^{-1}\right) / 2$, which carries the segment $-1 \leqq z \leqq 1$ counted twice into the unit circle $|w|=1$, and carries the ellipse $\gamma$ counted twice into the two circles $|w|=\rho,|w|=1 / \rho$. The first member of (10) can be written

$$
\frac{1}{2} \int_{|w|=1} w(z)\left|f(z)-P_{n}(z)\right| p\left(1-z^{2}\right)^{1 / 2}|d w|,
$$

so Theorem 1 applies (cf. WS for further details of the transformation and reasoning). A condition of form (3) on $|w|=\rho$ and $|w|=1 / \rho$ with respect to $w$ implies condition (3) as stated in Theorem 2, so the conclusion follows.

Another method of studying the situation of Theorem 2 is to avoid the transformation onto the w-plane, but to use the fact that for a polynomial $P(z)$ of degree $n$ the inequality $\int_{-1}^{1}|P(z)|{ }^{p} d z \leqq N^{p}, p>0$, implies $|P(z)| \leqq N N^{\prime} R^{n}$ on and within ${ }^{6} \gamma$. Here it is natural to assume that $w(z)$ itself is positive and continuous rather than $w(z)\left(1-z^{2}\right)^{1 / 2}$. We deduce as in the proof of Theorem 1 an inequality analogous to (9) giving the degree of convergence of the sequence $P_{n}(z)$ to $F(z)$ on $\gamma$; this degree of convergence then implies inequality (3) on $\gamma$, as we wish to prove. Thus we have proved the following theorem:

THEOREM 2a. Theorem 2 remains true if the requirement that $w(z)\left(1-z^{2}\right)^{1 / 2}$ is positive and continuous on $-1 \leqq z \leqq 1$ is replaced by the requirement that w(z) be positive and continuous on $-1 \leqq z \leqq 1$.

4. Approximation to a periodic function on the axis of reals. A different transformation of the plane will now yield a new result:

THEOREM 3. Let the function $f(z)$ be periodic with period $2 \pi$, and for $n=1,2, \ldots$ let there exist a trigonometric polynomial $t_{n}(z)$ of order $n$ such that we have

$$
\int_{-\pi}^{\pi} w(z)\left|f(z)-t_{n}(z)\right| p d z \leqq \frac{M}{\rho^{n p} n^{(k+\alpha+1) p}}, \quad 0<\alpha \leqq 1, p>0, \rho>1,
$$

where the weight function $w(z)$ is a positive, continuous function of period $2 \pi$, and $k$ is a nonnegative integer. Then if we define $F(z)=\lim _{n \rightarrow \infty} t_{n}(z)$, where $t_{n}(z)$ is still expressed as a trigonometric polynomial, the two functions $f(z)$ and $F(z)$ are equal almost everywhere

${ }^{6}$ Walsh, op. cit., Lemma, p. 92.

${ }^{7}$ Walsh and Sewell, this Bulletin, vol. 43 (1937), pp. 557-563. This method of proof of Theorem $2 \mathrm{a}$ is used widely in a number of similar situations in a forthcoming paper by the present writers, on "Problem $\beta$. " 
on the axis of reals. Moreover if $z=x+i y$, the function $F(z)$ is analytic for $|y|<\log \rho$, continuous in the corresponding closed region, and on the lines $y= \pm \log \rho$ satisfies an inequality of type (3).

Theorem 3 is a direct consequence of Theorem 1 , by virtue of the transformation $w=e^{i z}$, in the notation of Theorem 3 (compare WS, proof of Theorem 5).

5. Convergence properties of sequences. The methods of proof of Theorems 1, 2, and 3 apply also to the study of the degree of convergence of the sequences involved on various point sets not yet mentioned. Thus under the hypothesis of Theorem 1, the method already used shows that in the annulus $\rho_{1} \geqq|z| \geqq 1 / \rho_{1}, \rho_{1}<\rho$, we have instead of (7)

$$
\left|P_{n+1}(z, 1 / z)-P_{n}(z, 1 / z)\right| \leqq \frac{M_{2} \rho_{1}^{n}}{\rho^{n} n^{k+\alpha+1}} .
$$

Then for $\rho_{1} \geqq|z| \geqq 1 / \rho_{1}$ we may write

$$
\begin{aligned}
\left|F(z)-P_{n}(z, 1 / z)\right| \leqq & \left|P_{n+1}(z, 1 / z)-P_{n}(z, 1 / z)\right| \\
& +\left|P_{n+2}(z, 1 / z)-P_{n+1}(z, 1 / z)\right|+\cdots \\
\leqq & M_{2} \sum_{j=n}^{\infty} \frac{\rho_{1}}{\rho^{j} j^{k+\alpha+1}} \leqq \frac{M_{2}}{n^{k+\alpha+1}} \sum_{j=n}^{\infty}\left(\frac{\rho_{1}}{\rho}\right)^{j} \leqq \frac{M^{\prime} \rho_{1}^{n}}{\rho^{n} n^{k+\alpha+1}} .
\end{aligned}
$$

That is to say, we have established a further result:

COROLlary to THEOREM 1. Under the hypothesis of Theorem 1 we have in the annulus $\rho_{1} \geqq|z| \geqq 1 / \rho_{1}, \rho_{1}<\rho$,

$$
\left|F(z)-P_{n}(z, 1 / z)\right| \leqq \frac{M^{\prime} \rho_{1}^{n}}{\rho^{n} n^{k+\alpha+1}},
$$

where $M^{\prime}$ is a suitably chosen constant independent of $n$ and $z$.

The corollary just established implies corresponding statements connected with Theorems 2 and 3.

Corollary to Theorem 2 (or Theorem 2a). Under the hypothesis of Theorem 2 (or Theorem 2a) we have for $z$ on and within the ellipse whose foci are +1 and -1 and whose sum of semi-axes is $\rho_{1}<\rho$

$$
\left|F(z)-P_{n}(z)\right| \leqq \frac{M^{\prime} \rho_{1}^{n}}{\rho^{n} n^{k+\alpha+1}},
$$

where $M^{\prime}$ is a suitably chosen constant independent of $n$ and $z$. 
In the proof of the Corollary to Theorem 2a we employ the method of proof of Theorem 2a rather than the Corollary to Theorem 1 itself.

Corollary to TheORem 3. Under the hypothesis of Theorem 3 we have for $z$ in the region $|y|<\log \rho_{1}<\log \rho$

$$
\left|F(z)-t_{n}(z)\right| \leqq \frac{M^{\prime} \rho_{1}^{n}}{\rho^{n} n^{k+\alpha+1}},
$$

where $M^{\prime}$ is a suitably chosen constant independent of $n$ and $z$.

It may be noted that the inequalities representing the conclusions of these corollaries, with the obvious understanding that these functions $P_{n}(z, 1 / z), P_{n}(z), t_{n}(z)$ are polynomials of the kind considered of respective degree $n$, themselves imply the conclusions of Theorems 1, 2 (or 2a), and 3 (compare WS, Theorems 1, 3, and 5).

6. Reciprocal theorems. In the direction of a converse of Theorem 1 we indicate the proof of the following result:

THEOREM 4. Let the function $F(z)$ be analytic for $\rho>|z|>1 / \rho<1$, continuous in the corresponding closed region, and let $F^{(k)}(z), k$ a nonnegative integer, satisfy a Lipschitz condition 8 of order $\alpha$ on $|z|=\rho$ and $|z|=1 / \rho$. Let $p>0$ be given. Let $w(\theta)$ be a nonnegative function of $\theta$, periodic with period $2 \pi$, and Lebesgue-integrable for $-\pi \leqq \theta \leqq \pi$. Then there exists a sequence of polynomials $P_{n}(z, 1 / z)$ in $z$ and $1 / z$ of degrees $n=1,2, \cdots$ so that we have

$$
\int_{-\pi}^{\pi} w(\theta)\left|F(z)-P_{n}(z, 1 / z)\right| p d \theta \leqq \frac{M}{\rho^{n p} n^{(k+\alpha) p}},
$$

where $M$ is a suitably chosen constant independent of $n$ and $z$.

Theorem 4 is an immediate consequence of the corresponding theorem for Tchebycheff approximation (WS, Theorem 2). We add the remark that the conclusion of Theorem 4 , holding for some sequence $P_{n}(z, 1 / z)$, holds $a$ fortiori for the sequence $P_{n}(z, 1 / z)$ of best approximation in the sense of least $p$ th powers. The Corollary to Theorem 1 applies to the latter.

Corresponding results in the directions of converses of Theorems 2 , $2 \mathrm{a}$, and 3 can be proved with no less ease.

It will be noticed that there is a discrepancy of unity between the exponents of $n$ in Theorems 1 and 4. This discrepancy is not acci-

\footnotetext{
${ }^{8}$ That is to say, let (3) be satisfied with $\beta=0$.
} 
dental, but is inherent in the nature of the problem, as is shown by examples that the authors plan to publish elsewhere.

7. Generality of weight functions. The mere casual comparison of Theorems 2 and 2 a suggests that the particular restrictions we have placed on the weight functions are artificial rather than essential to the problem. The most general weight function that can be employed seems to be difficult to determine, both here and in the case of polynomial approximation purely in the real domain. Nevertheless we shall prove the following additional proposition:

THEOREM 5. Theorems 1, 2, 2a, and 3 persist if the requirement that the respective weight functions $w(\theta), w(z)\left(1-z^{2}\right)^{1 / 2}, w(z), w(z)$ be positive and continuous is replaced by the requirement that the weight functions be nonnegative, Lebesgue-integrable, and that some negative power of the corresponding weight functions be Lebesgue-integrable. ${ }^{9}$

The situation of Theorem 1 is typical. Under the new assumption of Theorem 5 we assume $[w(\theta)]^{-\beta}$ to be integrable in the interval $-\pi \leqq \theta \leqq \pi$, with $\beta>0$. The Hölder inequality

$$
\left|\int F^{\delta} G^{1-\delta}\right| \leqq\left(\int|F|\right)^{\delta}\left(\int|G|\right)^{1-\delta}, \quad 0<\delta<1,
$$

with $\delta=1 /(1+\beta)$, gives

$$
\begin{aligned}
\int_{-\pi}^{\pi} \mid f(\theta)- & s_{n}(\theta) \mid p(1-\delta) d \theta \\
& \leqq\left(\int_{-\pi}^{\pi} \frac{d \theta}{[w(\theta)]^{(1-\delta) / \delta}}\right)^{\delta}\left(\int_{-\pi}^{\pi} w(\theta)\left|f(\theta)-s_{n}(\theta)\right| p d \theta\right)^{1-\delta},
\end{aligned}
$$

and by virtue of (1) we have

$$
\int_{-\pi}^{\pi}\left|f(\theta)-s_{n}(\theta)\right|^{p(1-\delta)} d \theta \leqq \frac{M^{\prime}}{\rho^{n p(1-\delta)} n^{(k+\alpha+1) p(1-\delta)}} .
$$

This last inequality leads to an inequality similar to (6) and thence as before to the conclusion of Theorem 1 .

HARVARD UNIVERSITY AND

Georgia School of Technology

9 This condition on the weight functions has been previously used by Dunham Jackson in the study of approximation in the real domain, and by Walsh (op. cit., pp. 104-105) in the complex domain. 